\title{
Reisen mit chronisch entzündlichen Darmerkrankungen
}

\begin{abstract}
Bei Patienten mit einer stabil eingestellten chronisch entzündlichen Darmerkrankung (CED) spricht nichts gegen Reisen in nahe und ferne Länder, wenn sie vorher richtig beraten werden. Wichtig ist eine ausreichende Versorgung mit den notwendigen Medikamenten, auch im Falle eines entzündlichen Schubs.
\end{abstract}

Ihre Patienten mit CED sind reiselustig? Dann nur zu. Denn wenn Patienten mit Morbus Crohn oder Colitis ulcerosa auf Reisen gehen, verläuft dies nach Erfahrung von Professor Dr. Wolfgang Kruis, Köln, „erstaunlich unproblematisch“. Wichtig ist es, sie im Vorfeld gut zu beraten und mit der notwendigen Medikation auszustatten. Professor Dr. Stephan Vavricka, Zürich, sieht vor allem folgende Problemsituationen: Durch reisebedingten Stress, Nahrungsumstellung oder auch mangelnde Compliance im Reiseland kann sich die Erkrankung verschlechtern oder ein Schub ausgelöst werden. Zudem können endemische Infektionen, die bei Reisen nicht selten sind und mit Übelkeit und Durchfall einhergehen, unter einer immunsuppressiven Therapie schwerer verlaufen. Die Patienten sollten deshalb eine ausreichende Menge an Medikamenten zur Therapie ihrer CED im Gepäck haben. Nach entsprechender Aufklärung ist auch die Mitgabe von Steroiden zu überlegen. Vavricka empfahl zusätzlich die prophylaktische Verordnung von Antibiotika im Falle einer Gastroenteritis.

\section{Impfen unter Immunsuppression?}

Auch das Thema „Impfen“ kann, je nach Reiseland, relevant werden. Die Impfung mit Totimpfstoffen, etwa gegen Tetanus und Diphtherie ist auch unter Immunsuppression kein Problem. Attenuierte Lebendimpfstoffe dürfen dagegen unter einer immunsuppressiven Therapie nicht geimpft werden.

Zudem muss beachtet werden, dass Immunsuppressiva die Empfindlichkeit der Haut auf UV-Strahlung erhöhen. Nehmen die Patienten beispielsweise Azathioprin (z. B. Azafalk ${ }^{\circledR}$ ), Ciclosporin, Methotrexat oder Sulfasalazin ein, sollten sie bei einem Aufenthalt in der Sonne ihre Haut möglichst mit einem Sonnenschutzmittel mit Lichtschutzfaktor 50 schützen.

\section{Vor VTE schützen}

Bei langen Flug- oder Busreisen muss dem erhöhten Risiko für eine venöse Thromboembolie (VTE) in den Beinen Rechnung getragen werden. In einer Kohortenstudie mit knapp 9.000 Passagieren stieg das Risiko ab einer Flugdauer von vier Stunden deutlich an. Ein Aufenthalt im Flugzeug zwischen null und vier Stunden ging mit einem Risiko von 0,9 pro 100.000 Flügen einher. Bei Flügen zwischen acht und zwölf Stunden lag die

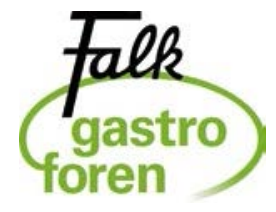

Besuchen Sie das nächste Falk Gastro Forum „Konservative und operative Therapien bei Erkrankungen von Leber und Ösophagus" am Samstag, den 28. Oktober 2017 in Dresden; Infos: www.falkfoundation.de/veranstaltungen

Gefahr bereits bei 15,8/100.000 und wer länger als zwölf Stunden in der Luft war hatte ein Risiko von 53/100.000 [Kuipers S et al. PLoS Med 2007; 4(9):e290]. Bei Patienten mit einer CED ist das Risiko für ein solches „economy class syndrome“ weiter erhöht, nämlich um das Zweifache gegenüber einer gesunden Kontrollgruppe [Fumery M et al. JCC 2014; 8(6):469479]. „Das Problem besteht vor allem bei Flügen ab einer Flugdauer von drei Stunden“, resümierte Vavricka. Dann sollte eine Thromboembolieprophylaxe überlegt werden.

\section{Hypoxie in höheren Regionen}

Wer hoch in die Luft geht, sei es mit dem Flugzeug, bei Wanderungen oder anderen Aufenthalten in höheren Regionen, ist einer Hypoxie ausgesetzt, die einen ungünstigen Effekt auf die entzündliche Darmerkrankung haben kann. Die Bedeutung der Hypoxie als beeinflussender oder auslösender Faktor einer Inflammation wurde in den letzten Jahren immer wieder diskutiert.

Vavricka konnte in aufwendigen Untersuchungen zeigen, dass Flüge und Aufenthalte in Höhen über 2.000 Metern über dem Meeresspiegel zu den Risikofaktoren bei Patienten mit chronischen entzündlichen Darmerkrankungen gehören und die entzündliche Aktivität der Erkrankung erhöhen können. Das gilt sowohl für Morbus Crohn als auch für Colitis ulcerosa [Vavricka SR et al. JCC 2014; 8(3):191-199]. Auch dies ist ein Grund, Patienten, die auf Reisen gehen, adäquat mit Medikamenten zu versorgen.

Dr. Beate Fessler

28. Interdisziplinäres Symposium Chronisch entzündliche Darmerkrankungen, Mannheim, 28. April 2017

(Veranstalter: Falk Foundation e.V.) 